OPEN ACCESS

Edited by:

Basem M. William,

The Ohio State University,

United States

Reviewed by:

Didier Blaise

Institut Paoli-Calmettes (IPC), France

Belinda Pinto Simoes,

University of São Paulo, Brazil

*Correspondence:

Qi Deng

kachydeng@126.com

Donglin Yang

yangdonglin@ihcams.ac.cn

Specialty section

This article was submitted to

Hematologic Malignancies,

a section of the journal

Frontiers in Oncology

Received: 01 July 2020

Accepted: 28 August 2020

Published: 29 September 2020

Citation:

Liu P, Liu M, Lyu C, LuW, Cui R,

Wang J, Li Q, Mou N, Deng Q and Yang D (2020) Acute

Graft-Versus-Host Disease After Humanized Anti-CD19-CAR T Therapy

in Relapsed B-ALL Patients After Allogeneic Hematopoietic Stem Cell

Transplant. Front. Oncol. 10:573822.

doi: 10.3389/fonc.2020.573822

\section{Acute Graft-Versus-Host Disease After Humanized Anti-CD19-CAR T Therapy in Relapsed B-ALL Patients After Allogeneic Hematopoietic Stem Cell Transplant}

\author{
Pengjiang Liu ${ }^{1}$, Meijing Liu ${ }^{2}$, Cuicui Lyu ${ }^{1}$, Wenyi Lu ${ }^{1}$, Rui Cui ${ }^{1}$, Jia Wang ${ }^{1}$, Qing Li ${ }^{1}$, \\ Nan Mou ${ }^{3}$, Qi Deng ${ }^{1 *}$ and Donglin Yang ${ }^{4 *}$ \\ ${ }^{1}$ Department of Hematology, Tianjin First Central Hospital, Tianjin, China, ${ }^{2}$ The First Central Clinical College of Tianjin Medical \\ University, Tianjin, China, ${ }^{3}$ Shanghai Genbase Biotechnology Co., Ltd., Tianjin, China, ${ }^{4}$ State Key Laboratory of Experimental \\ Hematology, National Clinical Research Center for Blood Diseases, Institute of Hematology and Blood Diseases Hospital, \\ Chinese Academy of Medical Sciences and Peking Union Medical College, Tianjin, China
}

We studied the acute graft-versus-host disease (GVHD) after humanized anti-CD19CAR T therapy in relapsed B-acute lymphoblastic leukemia (ALL) patients after allogeneic hematopoietic stem cell transplant (allo-HSCT). Fifteen B-ALL patients were enrolled in our study. Thirteen patients (86.67\%) achieved a complete response (CR) or CR with incomplete count recovery. The donor chimerism of the 13 patients reached $99.86 \pm$ $0.21 \%$. The development of aGVHD was observed in 10 patients (66.67\%). Six patients developed grade I-II of aGVHD, while the other four patients developed grade III-IV of aGVHD. The notable adverse events were grade 1-2 cytokine release syndrome (CRS) in 10 patients and grade 3-4 CRS in five patients. Two patients died of infection, while another patient died of sudden cardiac arrest. The anti-CD19-CAR T cells were not eliminated in peripheral blood when the patients developed aGVHD. However, we did not observe their expansion peaks again in the process of aGVHD. During the aGVHD, the peaks of IL-6 and TNF-a were correlated with aGVHD levels. By May 31, 2020, the rates of leukemia-free survival (LFS) and overall survival (OS) at 180 days were 53.846 and $61.638 \%$, respectively. All the patients who survived to date experienced aGVHD after humanized anti-CD19-CAR T cell therapy.

Trial registration: The patients were enrolled in clinical trials of ChiCTR-ONN-16009862 and ChiCTR1800019622.

Keywords: acute lymphoblastic leukemia, relapse, allogeneic hematopoietic stem cell transplantation, chimeric antigen receptor (CARs), graft-versus-host disease, cytokine release syndrome

\section{INTRODUCTION}

Allogeneic hematopoietic stem cell transplant (allo-HSCT) is an effective therapy for B-cell acute lymphoblastic leukemia (B-ALL). However, some patients relapse after allo-HSCT with a median survival of fewer than 6 months (1). Thus, treating these patients remains a challenge. Therapies for relapsed B-ALL patients after allo-HSCT 
include chemotherapy, donor lymphocyte infusion (DLI), and a second allo-HSCT. In all these salvage treatments, a low rate of remission and short survival has been evidenced (2). Also, therapies such as DLI and the second allo-HSCT can cause acute graft-versus-host disease (aGVHD), a severe and lethal immune response (3). Effective new treatment strategies for relapsed patients after allo-HSCT are urgently needed.

Anti-CD19 chimeric antigen receptor modified (anti-CD19CAR) $\mathrm{T}$ cell therapy has been effective eliminating malignant $\mathrm{B}$ cells (4). It has a high remission rate and long-term remission in patients with relapsed/refractory (R/R) B-ALL (5-7). Although this therapy is effective, it can cause severe toxicities. These toxicities include the cytokine release syndrome (CRS) and CAR-T cell-related encephalopathy syndrome (CRES), which are severe or even fatal (8). CRS is a complication associated with significantly elevated serum levels of inflammatory cytokines $(8,9)$.

Some studies have reported that donor-derived allogeneic anti-CD19-CAR T-cell therapy can effectively eradicate B-cell malignancies in B-ALL patients relapsing after allo-HSCT (10, 11). Allogeneic anti-CD19-CAR T-cell therapy can achieve a surprisingly good result without serious aGVHD in most of these research $(11,12)$.

In our clinical trial, we did not observe the mild aGVHD after anti-CD19-CAR T-cell therapy found in other studies. The anti-CD19-CAR T-cells used in this study were humanized anti-CD19-CAR $\mathrm{T}$ cells. It has been reported that patients can produce an immune response specific to murine $s c F v$ after murine CD19-CAR-T therapy, and exhibit a subsequent failure to murine CD19-CAR-T treatment (13). Taking advantage of humanized scFvs might reduce the immunogenicity of murine CD19 CAR-T cells and improve the longevity of anti-CD19-CAR $\mathrm{T}$ cells in patients (14). After the humanized anti-CD19-CAR T-cell therapy, the development of aGVHD was observed in 10 patients $(66.67 \%)$ of all 15 B-ALL patients who relapsed after allo-HSCT. Four patients developed grade III-IV aGVHD. The donors of these four patients were all haploid donors. However, all the patients who survived to date experienced aGVHD after humanized anti-CD19-CAR T cell therapy.

\section{PATIENTS AND METHODS}

\section{Participants in the Clinical Trial}

Fifteen B-cell acute lymphoblastic leukemia (ALL) patients who relapsed after allogeneic hematopoietic stem cell transplant (alloHSCT) between April 2018 and February 2020 were admitted to the Department of Hematology in Tianjin First Center Hospital (Tianjin, China). They all had high CD19 expression in malignant B cells analyzed by flow cytometry (FCM). They were enrolled in clinical trials of anti-CD19 chimeric antigen receptor modified (anti-CD19-CAR) T cell expressing humanized antiCD19 scFv and 4-1BB-CD3 $\zeta$ costimulatory-activation domains therapy (ChiCTR1800019622 and ChiCTR1800019622). All patients provided informed consent before enrollment. The final follow-up visit for endpoint analysis was conducted on May 31, 2020.

\section{The Source of T-Cells for Anti-CD19-CAR T-Cell Therapy}

Because the donor chimerism in peripheral blood in most patients was below $60 \%$, the donors of allo-HSCT provided peripheral blood mononuclear cells (PBMCs) for this anti-CD19CAR T-cell therapy.

\section{Generation and Detection of Anti-CD19-CAR T Cells and Transduction Efficiency}

PBMCs were collected and isolated by Ficoll density gradient centrifugation. CD3 + T cells were selected by CD3 microbeads (Miltenyi Biotec, Inc., Cambridge, MA, USA), stimulated by antiCD3/anti-CD28 mAb-coated Human T-Expander beads (Cat. no. 11141D; Thermo Fisher Scientific, Inc., Waltham, MA, USA) and cultured in T-cell medium X-Vivo 15 (Lonza Group, Ltd., Basel, Switzerland) supplemented with $250 \mathrm{IU} / \mathrm{mL}$ interleukin-2 (IL-2; Proleukin; Novartis International AG, Basel, Switzerland). All the CD3+ $\mathrm{T}$ cells $\left(3 \times 10^{6}\right)$ were transduced with a lentiviral vector encoding humanized CD19 CAR constructs (10 $\mu \mathrm{g}$, lenti-CD19-2rd-CAR; Shanghai Genbase Biotechnology Co., Ltd., Shanghai, CHINA) and cultured in media containing recombinant human IL-2 $(250 \mathrm{U} / \mathrm{ml})$. On the 12th day of cultivation, transduction efficiencies of anti-CD19-CAR were analyzed by flow cytometry (FCM) (BD Biosciences, San Jose, CA, USA).

\section{The lympho-Depleting Chemotherapy and Anti-CD19-CAR T-Cell Infusion}

All patients received lympho-depleting chemotherapy with fludarabine $\left(30 \mathrm{mg} / \mathrm{m}^{2}\right)$ and cyclophosphamide $\left(400 \mathrm{mg} / \mathrm{m}^{2}\right)$ from day -4 until day -2 . The humanized anti-CD19-CAR Tcells derived from their donors were infused $\left(1 \times 10^{6}\right.$ cells $\left./ \mathrm{kg}\right)$ on day 0 .

\section{Therapeutic Effect Observation and Donor Chimerism Analysis}

Therapy responses and minimal residual disease (MRD) analysis were assessed from day 14 post infusion. Disease status was defined as a complete response (CR), CR with incomplete count recovery (CRi), no remission (NR). Leukemia free survival (LFS) after the anti-CD19-CAR T-cell therapy was calculated from the date of $\mathrm{CR}$ to the date of disease recurrence. The changes in donor chimerism in bone marrow (BM) were analyzed using fluorescent-labeled multiple PCR amplification of short-tandem repeats (STR) analysis at the same time.

Disease status was defined as complete response (CR), CR with incomplete count recovery (Cri), or no remission (NR).

\section{Acute Graft-Versus-Host Disease (aGVHD) After Anti-CD19-CAR T-Cell Therapy}

The occurrence and extent of aGVHD were observed in the 15 patients from the day of anti-CD19-CAR T-cell infusion to the disappearance of aGVHD or death. Acute and chronic GVHD were classified using the Glucksberg and Seattle classical scales, respectively $(15,16)$. 
TABLE 1 | Patients baseline and therapy-related characteristics.

\begin{tabular}{|c|c|c|c|c|c|c|c|c|c|c|c|}
\hline Patient & Age & Diagnosis & Donor Type & $\begin{array}{l}\text { GVHD before } \\
\text { relapse }\end{array}$ & $\begin{array}{l}\text { Time from } \\
\text { transplant to } \\
\text { relapse }\end{array}$ & $\begin{array}{l}\text { Therapy after } \\
\text { relapse }\end{array}$ & $\begin{array}{l}\text { Blasts in BM } \\
\text { before CAR-T } \\
\text { Therapy (\%) }\end{array}$ & $\begin{array}{l}\text { Blasts in PB } \\
\text { before CAR-T } \\
\text { Therapy (\%) }\end{array}$ & $\begin{array}{l}\text { Donor } \\
\text { chimerism (BM) } \\
(\%)\end{array}$ & $\begin{array}{l}\text { Time from } \\
\text { relapse to } \\
\text { CAR-T therapy }\end{array}$ & $\begin{array}{l}\text { Time from stop } \\
\text { immuno- } \\
\text { suppressive } \\
\text { therapy to } \\
\text { CAR-T therapy }\end{array}$ \\
\hline Pt1\# & $41-45$ & B-ALL $(\mathrm{Ph}+)$ & MMUDT (8/10) & $\begin{array}{l}\text { Grade I aGVHD, } \\
\text { cGVHD }\end{array}$ & 58 months & TKI & 60.4 & 36.5 & 8.82 & 0.5 months & 51 months \\
\hline Pt 2\# & $46-50$ & B- ALL & MSDT & $\begin{array}{l}\text { Grade } \| \text { aGVHD, } \\
\text { cGVHD }\end{array}$ & 18 months & No & 26.2 & 16.4 & 20.55 & 0.5 months & 7 months \\
\hline Pt 3\# & $56-60$ & B-ALL & $\begin{array}{l}\text { Haplo-HSCT } \\
(7 / 10)\end{array}$ & Grade I aGVHD & 11 months & No & 5.6 & 2.6 & 79.12 & 1 months & 9 months \\
\hline Pt 4\# & $31-35$ & B-ALL & $\begin{array}{l}\text { Haplo-HSCT } \\
(5 / 10)\end{array}$ & No & 5 months & No & 68.8 & 46.5 & 39.35 & 0.5 months & 3 months \\
\hline Pt 5\# & $21-25$ & B-ALL & $\begin{array}{l}\text { Haplo-HSCT } \\
(5 / 10)\end{array}$ & $\begin{array}{l}\text { Grade I aGVHD, } \\
\text { cGVHD }\end{array}$ & 15 months & No & 45.2 & 22.9 & 48.36 & 0.5 months & 6 months \\
\hline Pt 6\# & $16-20$ & B-ALL & MUDT & Grade II aGVHD & 4 months & Two courses & 58.4 & 39.4 & 23.12 & 2 months & 3 months \\
\hline Pt 7\# & $11-15$ & B-ALL (Ph+) & $\begin{array}{l}\text { Haplo-HSCT } \\
(5 / 10)\end{array}$ & No & 6 months & No & 16.2 & 8.6 & 83.02 & 1 months & 5 months \\
\hline Pt 8\# & $11-15$ & B-ALL & MSDT & Grade I aGVHD & 14 months & One course & 32.8 & 16.8 & 77.13 & 1 months & 9 months \\
\hline Pt 9\# & $21-25$ & B-ALL (Ph+) & $\begin{array}{l}\text { Haplo-HSCT } \\
(5 / 10)\end{array}$ & No & 13 months & TKI & 48.6 & 33.5 & 36.18 & 0.5 months & 11 months \\
\hline Pt 10\# & $56-60$ & B-ALL & MSDT & Grade I aGVHD & 9 months & Two courses & 52.4 & 50.4 & 52.08 & 3 months & 4 months \\
\hline Pt 11\# & $21-25$ & B-ALL & MSDT & No & 24 months & No & 40.8 & 29.8 & 50.25 & 0.5 months & 20 months \\
\hline Pt 12\# & $16-20$ & B-ALL (Ph+) & $\begin{array}{l}\text { Haplo-HSCT } \\
(5 / 10)\end{array}$ & Grade I aGVHD & 9 months & TKI & 82.0 & 66.8 & 29.14 & 1 months & 4 months \\
\hline Pt 13\# & $21-25$ & B-ALL & $\begin{array}{l}\text { Haplo-HSCT } \\
(5 / 10)\end{array}$ & Grade II aGVHD & 15 months & Three courses & 12.8 & 8.8 & 85.16 & 6 months & 6 months \\
\hline Pt 14\# & $16-20$ & B-ALL & $\begin{array}{l}\text { Haplo-HSCT } \\
(5 / 10)\end{array}$ & Grade II aGVHD & 7 months & No & 40.2 & 25.5 & 56.75 & 0.5 months & 3 months \\
\hline Pt 15\# & $11-15$ & B-ALL & $\begin{array}{l}\text { Haplo-HSCT } \\
(5 / 10)\end{array}$ & Grade I aGVHD & 5 months & No & 65.5 & 45.6 & 42.47 & 0.5 months & 0.5 months \\
\hline
\end{tabular}

HLA-matched sibling donor transplantation, MSDT; HLA-matched unrelated donor transplantation, MUDT; HLA-mismatched unrelated donor transplantation, MMUDT; Haploid donor transplantation, Haplo-HSCT; Tyrosine kinase inhibitor, TKl.

Chemotherapy after relapse: Pt 6\# Two courses of VDCP. Pt 8\# One course of VCP. Pt 10\# One course of FLAG and one course of VDCP.

Pt 13\# Two courses of Hyper-CVAD and one course of FLAG. 


\section{Adverse Events and Anti-CD19-CAR T-Cell Expansion}

The side effects were observed from 0 to 14 days after CAR Tcell infusion. The cytokine release syndrome (CRS) was graded according to the adopted CRS scoring system and the National Cancer Institute Common Terminology Criteria for Adverse Events v4.03 after CAR T-cell infusion (17).

The secretion levels of cytokines, including interleukin-6 (IL6), IL-2R, tumor necrosis factor- $\alpha$ (TNF- $\alpha$ ), and IL-8 were measured on days $0,7,14,21$, and 28 by enzyme linked immunosorbent assay with a double-antibody one-step sandwich method. The proportions of CAR-T cells and the DNA level of the anti-CD19-CAR gene were detected around 110 days after antiCD19-CAR T-cell infusion. The expansion of anti-CD19-CAR $\mathrm{T}$-cells in CD3 $+\mathrm{T}$ cells in peripheral blood was observed using FCM. The DNA levels of the anti-CD19-CAR gene were detected by quantitative polymerase chain reaction (qPCR).

\section{Follow-Up}

A follow-up was done starting from the date of anti-CD19CAR T-cell infusion until the patients died. LFS was calculated from the date of CR/CRi after anti-CD19-CAR T-cell therapy to the date of relapse. OS was calculated from the date of CR after anti-CD19-CAR T-cell therapy to the date of death from any cause.

\section{Statistical Analysis}

Data were expressed as mean \pm SE. CRS was grouped and compared by Mann-Whitney rank and inspection. Non-normal distribution data are expressed as the median and interquartile range (IQR). Pearson correlation coefficient was used for evaluating the correlation between different factors. The LFS and OS probabilities were estimated with the Kaplan-Meier method and were compared using the log-rank test. All statistical analyses were computed using SPSS (version 17.0). $P<0.05$ were considered significant.

\section{RESULTS}

\section{Characteristics of the Patients in Our Study}

All patients enrolled in our study were B-ALL patients who relapsed after allo-HSCT. Reviewing their medical history, revealed that four patients (Pt 6\#, 8\#, 10\#, and 13\#) received chemotherapy after recurrence and before anti-CD19-CAR T-cell therapy. The detailed characteristics of all patients are shown in Table 1. The median proportion of leukemia cells was $43.73 \%$ (IQR 5.6-82.0) in BM and 30.01\% (IQR 2.6-66.8) in peripheral blood $(\mathrm{PB})$ when they were enrolled. The median proportion of donor chimerism in BM was $48.77 \%$ (IQR 8.82-85.16) when they were enrolled. The median time from relapse to CAR-T therapy was 1.27 (IQR 0.5-3.0) months. All patients had no GVHD when they enrolled in this clinical trial.

\section{The Transduction Efficiency and Immune Phenotype of Patients' T Lymphocytes}

The expression of CD3 + T cells in the culture system was $99.2 \%$ after screening with CD3 microbeads. The mean transduction

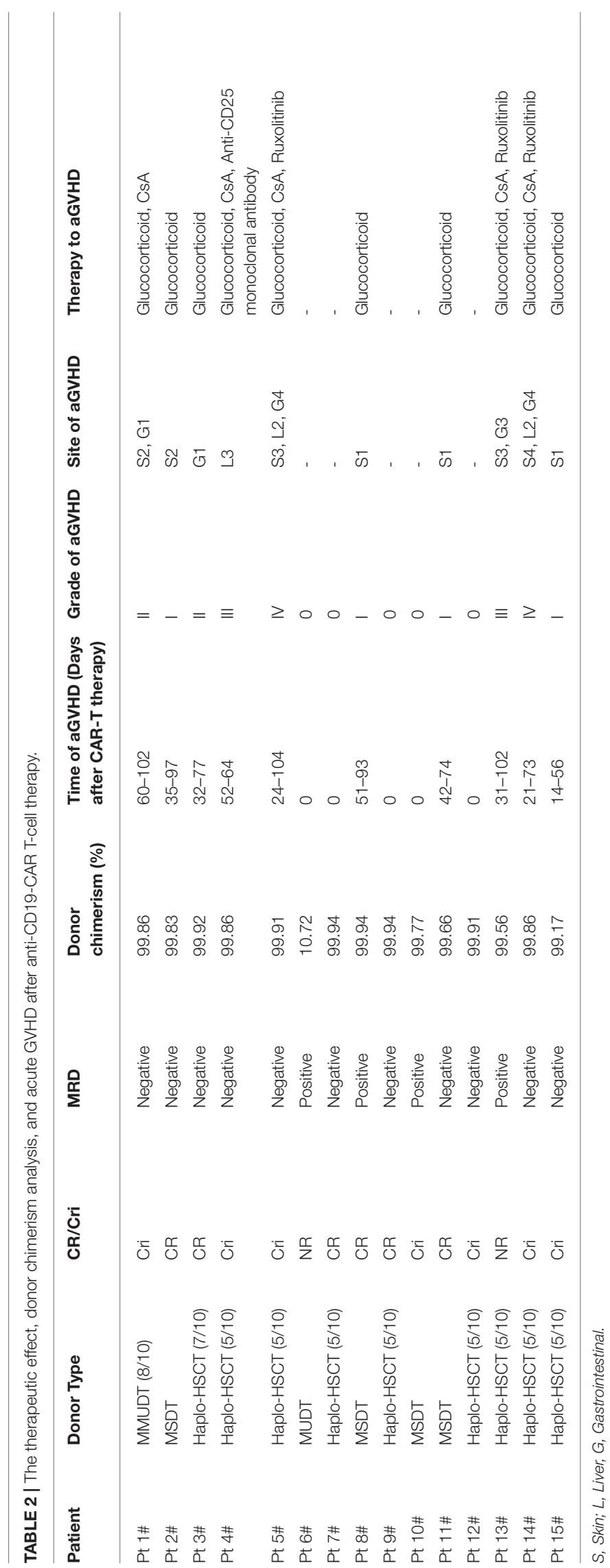


efficiency of the anti-CD19-CAR was $55.27 \pm 12.38 \%$. The mean humanized anti-CD19-CAR T-cells of all patients infused in this therapy was $1.26 \pm 0.36 \times 10^{6}$ cells $/ \mathrm{kg}$. The mean CD3 $+\mathrm{T}$ cells infused in this therapy was $2.28 \pm 0.61 \times 10^{6}$ cells $/ \mathrm{kg}$.

\section{Therapeutic Effect Observation and Donor Chimerism Analysis}

Fourteen days post anti-CD19-CAR T-cell infusion, 13 patients $(13 / 15,86.67 \%)$ achieved CR/CRi, six patients $(6 / 15,40.0 \%)$ achieved CR, seven patients $(7 / 15,46.67 \%)$ achieved CRi, and 11 patients $(11 / 15,73.33 \%)$ achieved MRD negative status. The Pt 6\# was evaluated as NR after this therapy. Because of the extramedullary leukemia of Pt 13\#, he did not achieve CR/CRi, even though there were no leukemia cells in his BM. Donor chimerism in BM rose to $99.81 \pm 0.20 \%$ in the 13 patients and achieved CR/Cri at 14 days after anti-CD19-CAR T-cell infusion. However, donor chimerism in BM of Pt 6\# was only $10.72 \%$, while donor chimerism in BM of Pt 13\# was $99.56 \%$ (Table 2).

\section{Acute GVHD After Anti-CD19-CAR T-Cell Therapy}

The development of aGVHD was observed in ten patients (10/15, $66.67 \%)$ in this group of B-ALL patients relapsed after alloHSCT. The donor type of these ten patients were haploid donors (Pt 3\#, 4\#, 5\#, 13\#, 14\#, 15\#), HLA-matched sibling donors (Pt 2\#, 8\#, 11\#), and HLA-mismatched unrelated donor (Pt 1\#). Six patients (Pt 1\#, 2\#, 3\#, 8\#, 11\#, and 15\#) $(6 / 15,40.0 \%)$ developed grade I-II of aGVHD from 14 days to 60 days post anti-CD19-CAR T-cell infusion. Four patients (Pt 4\#, 5\#, 13\#, and $14 \#)(4 / 15,26.67 \%)$ developed grade III-IV aGVHD from 21 days to 52 days post anti-CD19-CAR T-cell infusion. The other five patients $(5 / 15,33.33 \%)$ did not develop aGVHD. Donor selection, aGVHD occurrence and duration are shown in Table 2. Patients with grade I-II of aGVHD were controlled by glucocorticoid and cyclosporin A (CsA) therapy. Patients with grade III-IV of aGVHD were controlled by glucocorticoid, CsA, anti-CD25 monoclonal antibody and JAK1/JAK2 kinase inhibitor (Ruxolitinib) therapy. There were no aGVHD related deaths in our study.

\section{Adverse Events Observation of Anti-CD19-CAR T-Cell Therapy}

After the CAR-T cell infusion, patients manifested pyrexia with chills, accompanied by muscular weakness, fatigue, tachycardia, nausea, decreased appetite, increased serum alanine transaminase(ALT), glutamic oxalacetic transaminase(AST) and bilirubin, acute kidney injury and oliguria, electrolyte disturbance, and hematological toxicity (Table 3). Notable adverse events (AEs) in all 15 patients were grade 1-2 cytokine release syndrome (CRS) in ten patients $(10 / 15,66.67 \%)$ and grade $3-4$ CRS in five patients $(5 / 15,33.33 \%)$. No patients were diagnosed with CAR-T cell-related encephalopathy syndrome (CRES) in all the process of the treatment. No CRS or CRES related deaths occurred in our study. Fourteen of the 15 patients $(14 / 15,93.33 \%)$ developed grade $3-4$ hematological toxicity post
TABLE 3 | Adverse events observation of anti-CD19-CAR T-cell therapy.

Events

\section{General condition}

Temperature $\geq 38^{\circ} \mathrm{C}$ (fever)

Chills

Muscular weakness

Rash

Systolic blood pressure $<90 \mathrm{~mm} \mathrm{Hg}$ (hypotension)

Needing oxygen for $\mathrm{SaO}_{2}>90 \%$ (hypoxia)

Fatigue

Weight loss

The incidence

Organ toxicities

Cardiac

Tachycardia

Arrhythmias

Heart block

Respiratory

Hypoxia

Dyspnea

Cough

Pleural effusion

Gastrointestinal

Nausea

Vomiting

Decreased appetite

$13 / 15(86.67 \%)$ $6 / 15(40.00 \%)$

$10 / 15(66.67 \%)$

$2 / 15(13.33 \%)$

$1 / 15(6.67 \%)$

$2 / 15(13.33 \%)$

$11 / 15(73.33 \%)$

$4 / 15(26.67 \%)$

\section{Hepatic}

Increased serum ALT, AST

Increased serum bilirubin levels

Renal

Acute kidney injury (increased serum creatinine levels)

Oliguria

\section{$6 / 15(40.00 \%)$ \\ $1 / 15(6.67 \%)$ \\ $0 / 15(0 \%)$}

$3 / 15(20.00 \%)$

2/15 (13.33\%)

$5 / 15$ (33.33\%)

$2 / 15(13.33 \%)$

$7 / 15(46.67 \%)$

$3 / 15(20.00 \%)$

$8 / 15(53.33 \%)$

The electrolyte

Hypokalaemia

Hypocalcaemia

Hyperglycaemia

8/15 (53.33\%)

5/15 (33.33\%)

Coagulopathy

Disseminated intravascular coagulation

Neurological

Encephalopathy

$6 / 15(40.00 \%)$

$5 / 15(33.33 \%)$

Confused state

$7 / 15(46.67 \%)$

$7 / 15(46.67 \%)$

$4 / 15$ (26.67\%)

Dizziness

Aphasia

Somnolence

$1 / 15(6.67 \%)$

$0 / 15(0 \%)$

$0 / 15(0 \%)$

$2 / 15$ (13.33\%)

$0 / 15(0 \%)$

$3 / 15$ (20.00\%)

\section{Hematological}

Neutropenia (grade 3/4) $\left(<1^{*} 10^{9} / L\right)$

$14 / 15(93.33 \%)$

Anemia (grade $3 / 4)(<80 \mathrm{~g} / \mathrm{L}$ )

$12 / 15(80.00 \%)$

Thrombocytopenia (grade 3/4) $\left(<50^{*} 10^{9} /\right.$ L)

$14 / 15(93.33 \%)$

Cytokine release syndrome (CRS)

Grade 0 CRS

0/15 (0\%)

Grade 1 CRS

5/15 (33.33\%)

Grade 2 CRS

$5 / 15(33.33 \%)$

Grade 3 CRS

$3 / 15$ (20.00\%)

Grade 4 CRS

$2 / 15$ (13.33\%)

Grade 5 CRS 

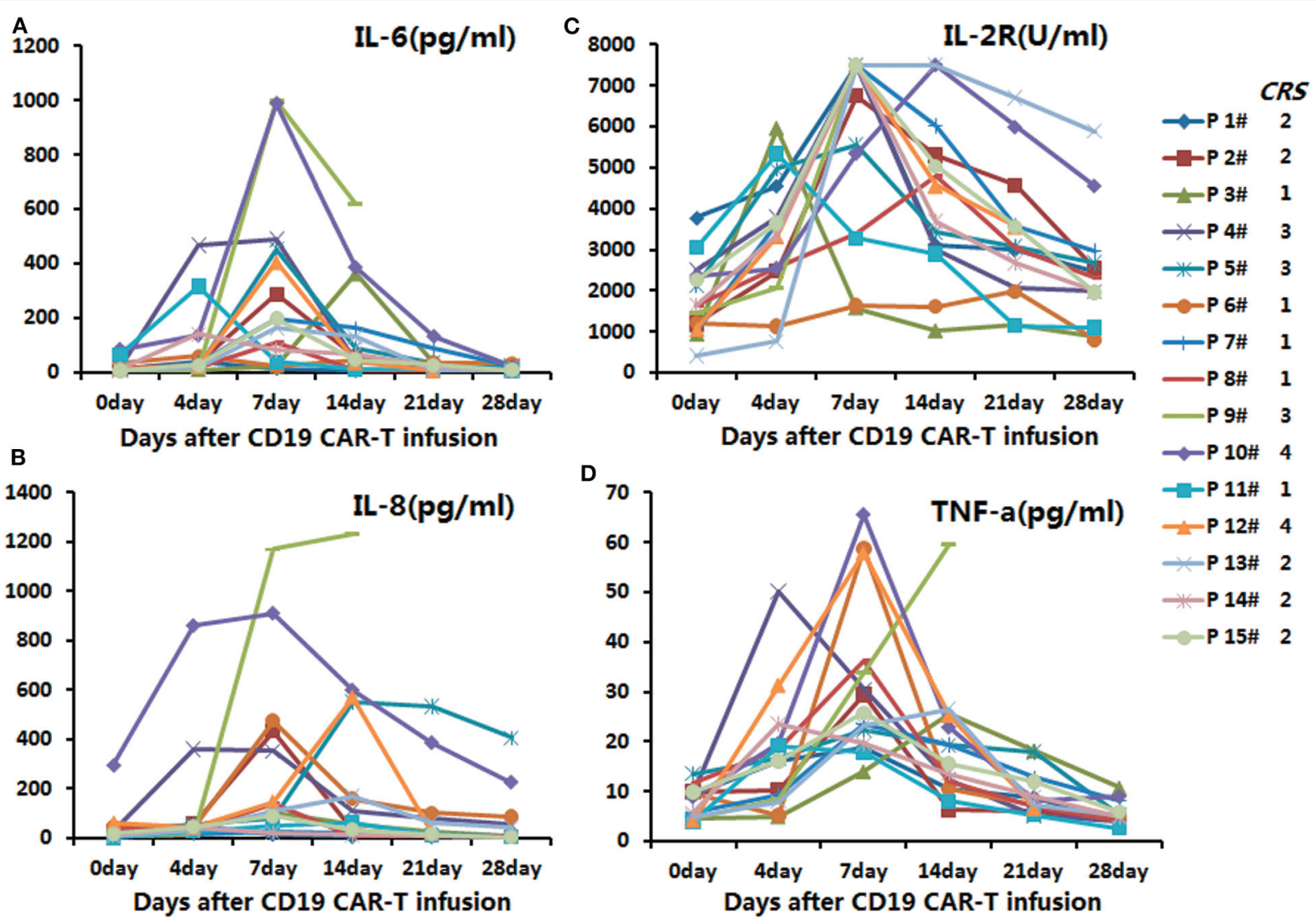

FIGURE 1 | The secretion levels of IL-6, IL-2R, TNF- $\alpha$, and IL-8 in the anti-CD19-CAR T-cell therapy. The peaks of the cytokines were on days 4-7 post infusion of anti-CD19-CAR T-cells, and the levels then declined 14 days post infusion. (A) The secretion levels of IL-6 in the anti-CD19-CAR T-cell therapy. (B) The secretion levels of IL-8 in the anti-CD19-CAR T-cell therapy. (C) The secretion levels of IL-2R in the anti-CD19-CAR T-cell therapy. (D) The secretion levels of TNF-a in the anti-CD19-CAR T-cell therapy.

anti-CD19-CAR T-cell infusion. The other patient developed grade 2 hematological toxicity only (Table 3 ).

In the anti-CD19-CAR T-cell therapy, the cytokines' peaks occurred on days four to seven post infusion of anti-CD19-CAR T-cells, and declined 14 days after the infusion (Figure 1).

All patients were given antipyretic drugs and methylprednisolone to overcome the AEs. Only the patients 10\# and $12 \#$, who developed grade 4 CRS were given tocilizumab during therapy. The AEs of the other patients were relieved 9-16 days post infusion.

\section{Expansion of Anti-CD19-CAR T-Cells and Anti-CD19-CAR Gene Expression}

The peak proportions of CAR-T cells in CD3 $+\mathrm{T}$ cells in peripheral blood were detected in the process of the anti-CD19CAR T cell therapy. The average expansion peak of anti-CD19CAR T cells was $32.92 \pm 20.44 \%$ on days 4 to 14 (median time was $11.31 \pm 3.47$ days) after the infusion (Figure 2A). The mean level of anti-CD19-CAR T cells was $1.98 \pm 1.54 \%$ when the patients developed aGVHD. We did not observe the expansion peak of anti-CD19-CAR T cells or anti-CD19-CAR gene expression again in the process of aGVHD. The DNA levels of anti-CD19CAR genes showed the same trend (Figure 2B).

\section{Correlation Analysis Between the aGVHD and Cytokine Levels, CAR-T Cell Levels, and T Cell Subsets}

All the cytokines' peaks occurred before 14 days post CAR$\mathrm{T}$ cell infusion, while the AEs of the patients had relieved 16 days post infusion, and all aGVHD cases occurred after 21 days post infusion. The 15 patients were divided into grade 0 -II of aGVHD and grade III-IV of aGVHD groups. The cytokine levels in the peripheral blood of patients receiving antiCD19-CAR T-cell therapy were uncorrelated with aGVHD levels (Figures 3A-D). During the occurrence of aGVHD, the peaks of IL-6 and TNF-a in grade I-II of aGVHD were lower than in grade III-IV of aGVHD (Figures 3E,H). The peaks of IL-2R and IL-8 during the occurrence of aGVHD were uncorrelated with aGVHD levels (Figures 3F,G). In all patients, the peaks of anti-CD19-CAR $T$ cells and anti-CD19-CAR gene expression in the peripheral blood were uncorrelated with aGVHD levels (Figures 3I,J). The anti-CD19-CAR T cells and anti-CD19-CAR 

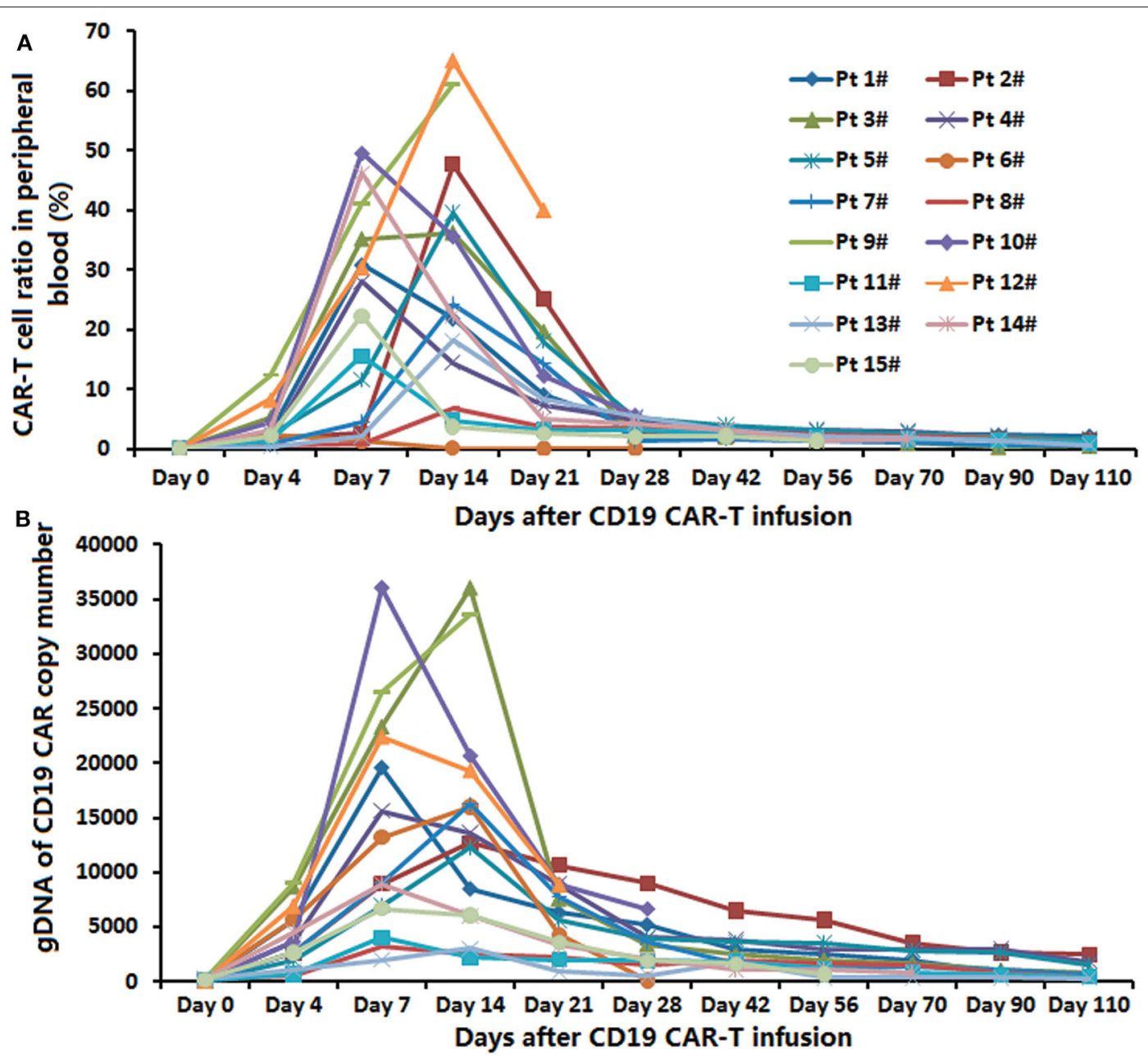

FIGURE 2 | Expansion of anti-CD19-CAR T-cells and anti-CD19-CAR gene. (A) The average expansion peak of anti-CD19-CAR T cells was $32.54 \pm 19.94 \%$ on days 4-14 (median time was $11.31 \pm 3.47$ days) after the infusion. The mean level of anti-CD19-CAR T cells was $2.58 \pm 1.32 \%$ when the patients developed aGVHD. (B) DNA level of anti-CD19-CAR gene showed the same trend.

gene expression changes during the occurrence of aGVHD were uncorrelated with aGVHD levels (Figures 3K,L). The $\mathrm{CD} 3+\mathrm{CD} 8+\mathrm{CD} 4$ - percentages in peripheral blood in grade III of aGVHD patients were lower than those of grade III-IV of aGVHD patients (Figure 3N). However,CD3+ percentage, $\mathrm{CD} 3+$ absolute value, and $\mathrm{CD} 3+\mathrm{CD} 8+\mathrm{CD} 4$ - absolute value during the occurrence of aGVHD were uncorrelated with the aGVHD levels (Figures 3M-P).

\section{Follow-Up}

The median follow-up time of the 15 patients was 258.69 days (15-660 days). By May 31, 2020, the rates of LFS and OS during 180 days were 53.846 and $61.638 \%$, respectively (Figures 4A,B). Pt 9\# died of sudden cardiac death 15 days post infusion, Pt $10 \#$ and 12\# died of serious infection at 21 and 24 days post infusion, respectively, when they had achieved CRi and were relieved from the AEs of this therapy. Pt 6\# and Pt 13\#, who had not achieved CR/CRi from this therapy died of disease progression 35 and 181 days post infusion, respectively. The Pt
7\# and Pt 8\# developed recurrence again at 75 and 103 days after the anti-CD19-CAR T cell infusion, respectively, and died 125 and 227 days after infusion, respectively. Figure 4 shows the LFS, OS, the occurrence of aGVHD, the cause of death and the CD19 expression at the monent of recurrence up to January 31, 2020.All patients who survived experienced aGVHD after antiCD19-CAR T cell therapy. In particular, the LFS of Pt $1 \#$ to Pt 5\# and Pt $11 \#$ was 480, 512, 660, 536, 421, and 183 days, respectively.

\section{DISCUSSION}

Allo-HSCT therapy is an effective treatment to improve the survival of B-ALL patients. However, several B-ALL patients with high-risk factors are still at a high risk of relapse after alloHSCT and poor long-term survival (18-20). Recurrence after allo-HSCT was the main cause of death after allo-HSCT (21). The B-ALL patients relapsed after allo-HSCT had a median survival of only 5.5 months (1). DLI with or without chemotherapy, 

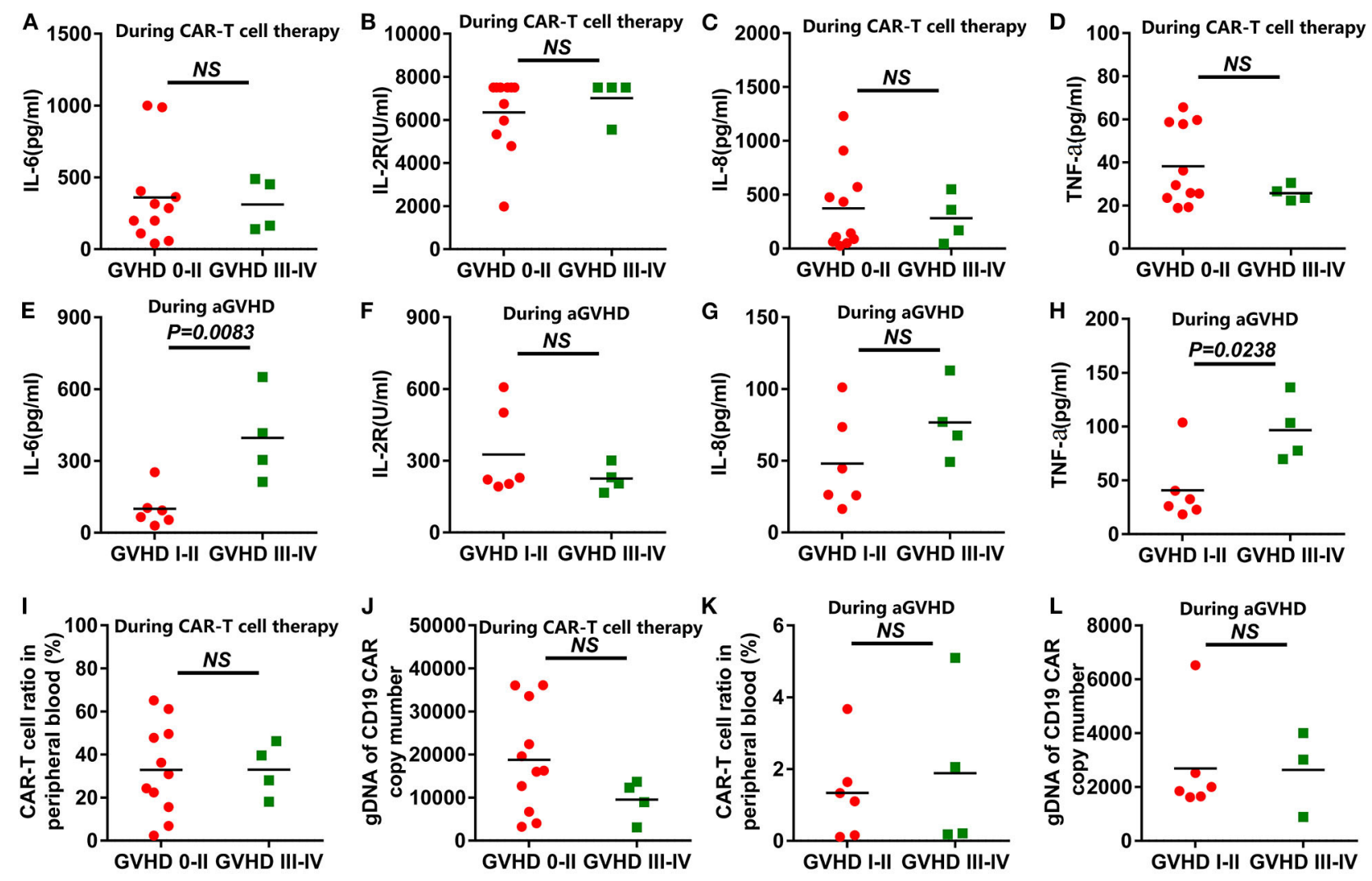

M

$\mathbf{N}$

O
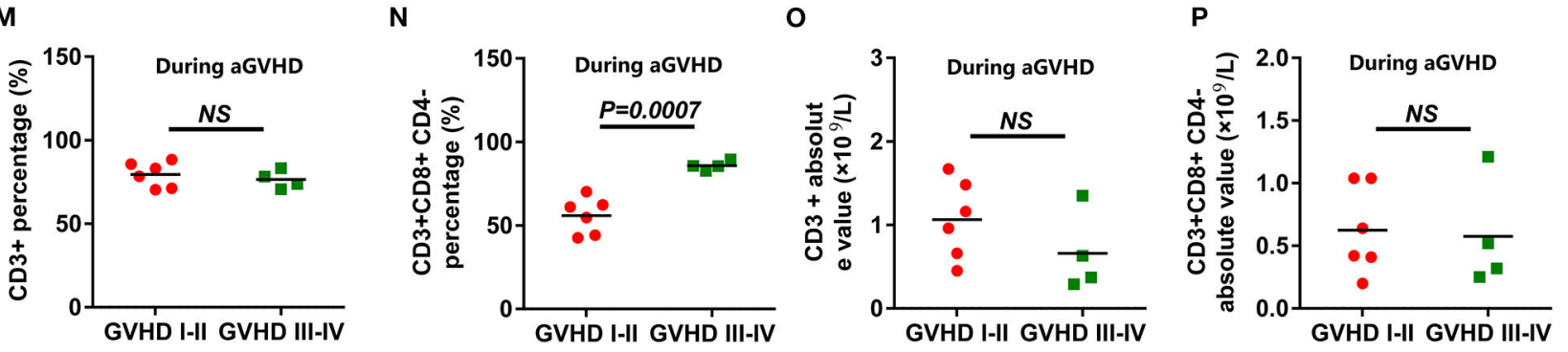

FIGURE 3 | Correlation analysis between the aGVHD and the cytokine levels or CAR-T cell levels. (A-D) The peaks of the cytokines in the anti-CD19-CAR T-cell therapy were uncorrelated with aGVHD levels. (E,H) The peaks of IL-6 and TNF-a in grade I-II in the aGVHD group were lower than those of the grade III-IV in the aGVHD group during the occurrence of aGVHD. (F,G) The peaks of the IL-2R and IL-8 during the occurrence of aGVHD were uncorrelated with aGVHD levels. (I-L) The peaks of anti-CD19-CAR T cells and anti-CD19-CAR gene were uncorrelated with aGVHD levels during the anti-CD19-CAR T-cell therapy and the occurrence of aGVHD. (N) The CD3+CD8+CD4- percentages in grade I-II in the aGVHD group were lower than that of the grade III-IV in the aGVHD group. (M,O,P) The CD3+ percentage, the CD3+ absolute value and the CD3+CD8+CD4- absolute value were uncorrelated with aGVHD levels during the occurrence of aGVHD.

is essential therapy to B-ALL patients who relapsed after alloHSCT. However, Only some patients responded to DLI therapy, and these patients had an increased risk of aGVHD which can be caused by the infusion of a large number of donor $\mathrm{T}$ cells (22-24). Thus, a $20 \%$ treatment-related mortality or the lack of response to it resulted in a median survival period of 6 months for the B-ALL patients that relapsed after allo$\operatorname{HSCT}(3)$.

Anti-CD19-CAR T-cell therapy had significant clinical efficacy in relapsed/refractory B-ALL patients who had not received allo-HSCT (25). An early study focused on donor antiCD19-CAR T-cell therapy for such patients (10). The anti-CD19CAR T-cell therapy derived from donor $\mathrm{T}$ cells was a selection of salvage therapy to B-ALL patients who relapsed after alloHSCT, because allogeneic anti-CD19-CAR T-cells exerted a graft versus leukemia(GVL) effect $(11,26-28)$. In these studies, donor anti-CD19-CAR T-cell therapy resulted in a high remission rate compared with conventional therapeutic approaches such as DLI. Two reviews summarized the effects and side effects of this therapy $(29,30)$. It was effective for minimal residual disease without serious side-effects, including aGVHD, CRS, and CRES. Therefore, effective and low toxicity anti-CD19-CAR Tcell therapy can be an option to overcome the poor prognosis of B-ALL patients who relapsed after allo-HSCT. However, the long-term efficacy and survival time for this therapy are not mentioned sufficiently. 

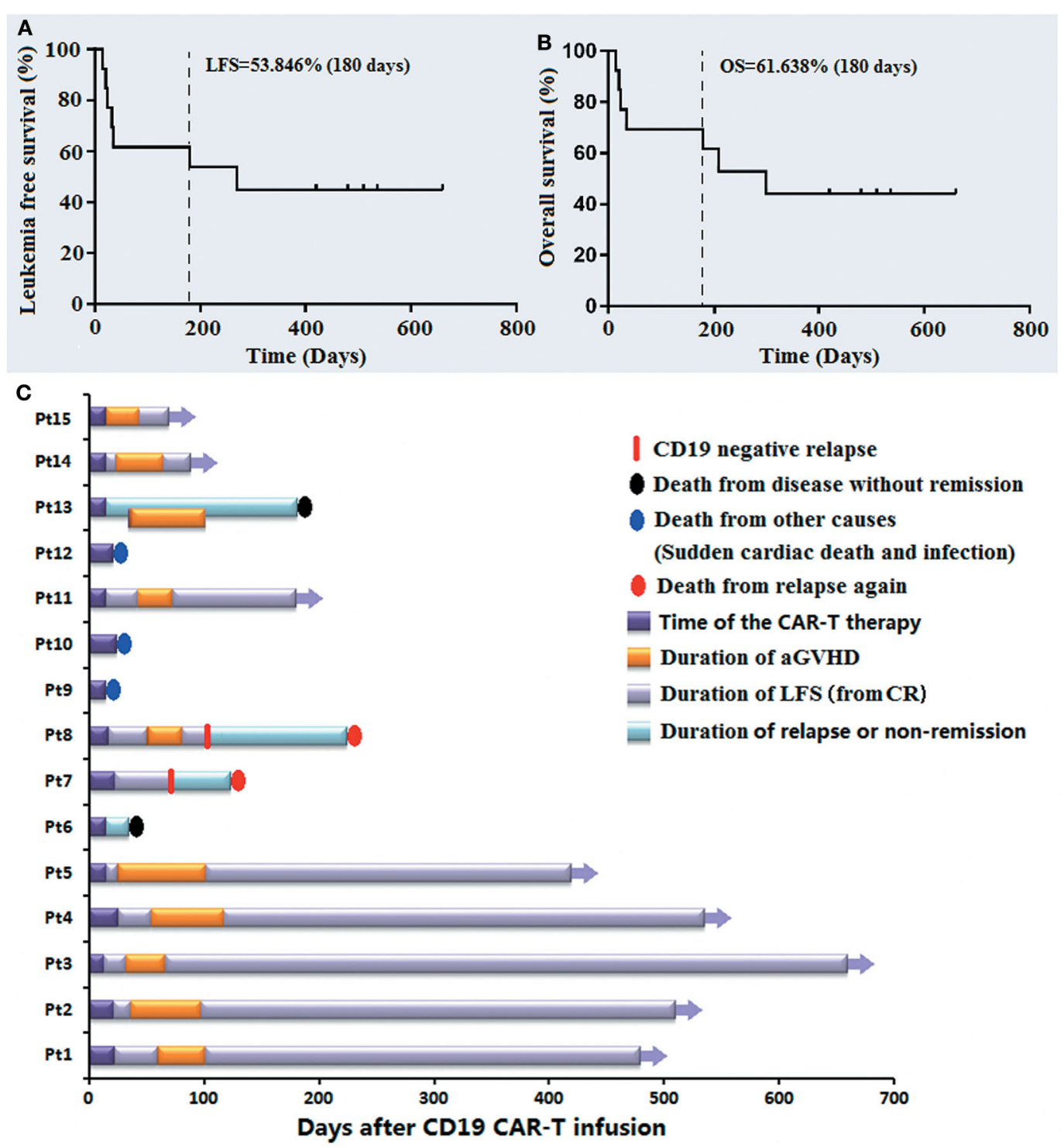

FIGURE 4 | The follow-up post anti-CD19-CAR-T therapy of the B-ALL patients who relapsed after allo-HSCT. (A,B) The LFS and OS of the 13 patients on 180 days were 53.846 and $61.638 \%$. (C) The LFS, OS, occurrence of aGVHD, cause of death and CD19 expression at the moment of recurrence are listed.

In our study, 13 patients (86.67\%) achieved CR/CRi 14 days after the anti-CD19-CAR T-cell infusion, including six patients (40.0\%) who achieved CR. Ten patients (66.67\%) achieved CR 28 days after infusion. Ten patients (66.67\%) developed grade 1-2 CRS, while five patients (33.33\%) developed grade $3-4$ CRS. The side-effects observed in our study were more serious than those in previous the studies $(10,11,26-28)$. The high median proportion of leukemia cells in BM (43.73\%) before enrollment can be one of the possible reasons for these serious side-effects. The development of aGVHD was observed in 10 patients $(66.67 \%)$ in this group. Six patients developed grade I-II of aGVHD, while four patients developed grade III-IV of aGVHD. The grades of aGVHD in our study were higher than those in previous studies $(10,11,26-28)$.

In previous studies, the reason for mild aGVHD after antiCD19-CAR T-cell therapy was unclear. The risk of developing
aGVHD after the anti-CD19-CAR T-cell infusion was different from the DLI infusion $(31,32)$. One explanation was that the dose of anti-CD19-CAR T-cell infusion was smaller than the dose of DLI. In our study, the mean anti-CD19-CAR Tcells infused in this therapy was $1.26 \pm 0.36 \times 10^{6}$ cells $/ \mathrm{kg}$ and the mean $\mathrm{CD} 3+\mathrm{T}$ cells infused was $2.28 \pm 0.61 \times$ $10^{6}$ cells $/ \mathrm{kg}$. Another explanation is that the persistence of anti-CD19-CAR T-cell was limited to $<4$ weeks in previous studies. In general, aGVHD occurred with a median of 4 weeks after the infusion of DLI. The mean level of antiCD19-CAR $\mathrm{T}$ cells was $1.98 \pm 1.54 \%$ when the patients developed aGVHD in this group of patients. The antiCD19-CAR $T$ cells were not eliminated from the patient at this time.

The anti-CD19-CAR T-cells used in our study were humanized anti-CD19-CAR $\mathrm{T}$ cells. HLA restricted T-cell 
mediated immune response to epitopes derived from murine scFv, which can affect the survival time of anti-CD19-CAR T-cells in vivo (33). Humanized anti-CD19-CAR $\mathrm{T}$ cells in our study can reduce the immunogenicity of murine CD19 CAR-T cells and prolong the survival time of cells in patients (34). Tumor burden was another critical factor that can influence the expansion of anti-CD19-CAR-T cells during this therapy $(13,35,36)$. It can be another factor that contributes to the longer existential time of antiCD19-CAR-T cells in our study. The last factor was that the donors of the four patients who developed grade IIIIV of aGVHD were all haploid donors. Whether these factors are the reasons for the higher rate of aGVHD in this group of patients, needs to be expanded using more case-studies.

In our clinical trial, we did not observe mild aGVHD after the anti-CD19-CAR T-cell therapy in previous studies. However, the AEs and aGVHD in our study were serious but controllable. Patients who had an extended survival time developed aGVHD after this treatment. In particular, five patients had an LFS for more than 400 days after the anti-CD19-CAR T-cell therapy and subsequent aGVHD.

\section{DATA AVAILABILITY STATEMENT}

All datasets generated for this study are included in the article/supplementary material.

\section{REFERENCES}

1. Spyridonidis A, Labopin M, Schmid C, Volin L, Yakoub-Agha I, Stadler M, et al. Outcomes and prognostic factors of adults with acute lymphoblastic leukemia who relapse after allogeneic hematopoietic cell transplantation. An analysis on behalf of the acute leukemia working party of ebmt. Leukemia. (2012) 26:1211-7. doi: 10.1038/leu.2011.351

2. Forman SJ, Rowe JM. The myth of the second remission of acute leukemia in the adult. Blood. (2013) 121:1077-82. doi: 10.1182/blood-2012-08-234492

3. Scarisbrick JJ, Dignan FL, Tulpule S, Gupta ED, Kolade S, Shaw B, et al. A multicentre UK study of GVHD following DLI: rates of GVHD are high but mortality from GVHD is infrequent. Bone Marrow Transplant. (2015) 50:62-7. doi: 10.1038/bmt.2014.227

4. Campana D, Schwarz H. Imai C. 4-1BB chimeric antigen receptors. Cancer J. (2014) 20:134-40. doi: 10.1097/PPO.0000000000000028

5. Park JH, Rivière I, Gonen M, Wang X, Sénéchal B, Curran KJ, et al. Long-term follow-up of CD19 CAR therapy in acute lymphoblastic leukemia. $N$ Engl J Med. (2018) 378:449-59. doi: 10.1056/NEJMoa1709919

6. Lee DW, Kochenderfer JN, Stetler-Stevenson M, Cui YK, Delbrook C, Feldman SA, et al. T cells expressing CD19 chimeric antigen receptors for acute lymphoblastic leukaemia in children and young adults: a phase 1 dose-escalation trial. Lancet. (2015) 385:517-28. doi: 10.1016/S0140-6736(14)61403-3

7. Maude SL, Laetsch TW, Buechner J, Rives S, Boyer M, Bittencourt H, et al. Tisagenlecleucel in children and young adults with b-cell lymphoblastic leukemia. N Engl J Med. (2018) 378:439-48. doi: 10.1056/NEJMoa1709866

8. Neelapu SS, Tummala S, Kebriaei P, Wierda W, Gutierrez C, Locke FL, et al. Chimeric antigen receptor T-cell therapy assessment and management of toxicities. Nat Rev Clin Oncol. (2018) 15:47-62. doi: 10.1038/nrclinonc. 2017.148

\section{ETHICS STATEMENT}

The studies involving human participants were reviewed and approved by Tianjin First Center Hospital (Tianjin, China). The patients/participants provided their written informed consent to participate in this study.

\section{AUTHOR CONTRIBUTIONS}

QD and DY: conception and design and study supervision. PL: drafting or reviewing of the manuscript. ML, CL, WL, RC, QL, and NM: acquisition of data. JW: analysis and interpretation of data. All authors: writing and review of manuscript.

\section{FUNDING}

The National Natural Science Foundation of China (81900186 and 81800105). The Non-profit Central Research Institute Fund of Chinese Academy of Medical Sciences. CAMS Innovation Fund for Medical Sciences (CIFMS, 2016-I2M-3023). Chun Miao Foundation of the First Central Hospital of Tianjin (2019CM04).

\section{ACKNOWLEDGMENTS}

We thank patients for their participation in our experimental studies and clinical trials. We thank the Shanghai Genbase Biotechnology Co., Ltd. for providing us with anti-CD19-CAR T-cells and technical support.

9. Maude SL, Pulsipher MA, Boyer MW, Grupp SA, Davies SM, Phillips CL, et al. Efficacy and safety of ctl019 in the first us phase ii multicenter trial in pediatric relapsed/refractory acute lymphoblastic leukemia: results of an interim analysis. Blood. (2016) 128:2801. doi: 10.1182/blood.V128.22.2801.2801

10. Kochenderfer JN, Dudley ME, Carpenter RO, Kassim SH, Rose JJ, Telford WG, et al. Donor-derived CD19-targeted T cells cause regression of malignancy persisting after allogeneic hematopoietic stem cell transplantation. Blood. (2013) 122:412939. doi: 10.1182/blood-2013-08-519413

11. Brudno JN, Somerville RPT, Shi V, Rose JJ, Halverson DC, Fowler DH, et al. Allogeneic T cells that express an anti-CD19 chimeric antigen receptor induce remissions of B-Cell malignancies that progress after allogeneic hematopoietic stem-cell transplantation without causing graft-versus-host disease. J Clin Oncol. (2016) 34:1112-21. doi: 10.1200/JCO.2015.64.5929

12. Chen Y, Cheng Y, Suo P, Yan C, Wang Y, Chen Y, et al. Donor-derived CD19-targeted $\mathrm{T}$ cell infusion induces minimal residual disease-negative remission in relapsed B-cell acute lymphoblastic leukaemia with no response to donor lymphocyte infusions after haploidentical haematopoietic stem cell transplantation. Br J Haematol. (2017) 179:598-605. doi: 10.1111/bjh.14923

13. Turtle CJ, Hanafi L-A, Berger C, Gooley TA, Cherian S, Hudecek M, et al. CD19 CAR-T cells of defined CD4+:CD8+ composition in adult B cell all patients. J Clin Invest. (2016) 126:2123-38. doi: 10.1172/JCI85309

14. Johnson LA, Scholler J, Ohkuri T, Kosaka A, Patel PR, McGettigan SE, et al. Rational development and characterization of humanized anti-EGFR variant III chimeric antigen receptor T cells for glioblastoma. Sci Transl Med. (2015) 7:275ra22. doi: 10.1126/scitranslmed.aaa4963

15. Glucksberg H, Storb R, Fefer A, Buckner CD, Neiman PE, Clift RA, et al. Clinical manifestations of graft-versus-host disease in human recipients of marrow from HL-A-matched sibling donors. Transplantation. (1974) 18:295304. doi: 10.1097/00007890-197410000-00001 
16. Shulman HM, Sullivan KM, Weiden PL, McDonald GB, Striker GE, Sale GE, et al. Chronic graft-versus-host syndrome in man. A long-term clinicopathologic study of 20 seattle patients. Am J Med. (1980) 69:20417. doi: 10.1016/0002-9343(80)90380-0

17. Lee DW, Gardner R, Porter DL, Louis CU, Ahmed N, Jensen M, et al. Current concepts in the diagnosis and management of cytokine release syndrome. Blood. (2014) 124:188-95. doi: 10.1182/blood-2014-05-552729

18. Pavletic SZ, Kumar S, Mohty M, Lima M, Foran JM, Pasquini M, et al. NCI First international workshop on the biology, prevention, and treatment of relapse after allogeneic hematopoietic stem cell transplantation: report from the committee on the epidemiology and natural history of relapse following allogeneic cell transplantation. Biol Blood Marrow Transplant. (2010) 16:87190. doi: 10.1016/j.bbmt.2010.04.004

19. Gökbuget N, Stanze D, Beck J, Diedrich H, Horst H-A, Hüttmann A, et al. Outcome of relapsed adult lymphoblastic leukemia depends on response to salvage chemotherapy, prognostic factors, and performance of stem cell transplantation. Blood. (2012) 120:2032-41. doi: 10.1182/blood-2011-12-399287

20. O’Brien S, Schiller G, Lister J, Damon L, Goldberg S, Aulitzky W, et al. High-dose vincristine sulfate liposome injection for advanced, relapsed, and refractory adult philadelphia chromosome-negative acute lymphoblastic leukemia. J Clin Oncol. (2013) 31:676-83. doi: 10.1200/JCO.2012.46.2309

21. Van den Brink MR, Porter DL, Giralt S, Lu SX, Jenq RR, Hanash A, et al. Relapse after allogeneic hematopoietic cell therapy. Biol Blood Marrow Transplant. (2010) 16(1 Suppl.):S138-45. doi: 10.1016/j.bbmt.2009.10.023

22. Castagna L,Sarina B,Bramanti S, Perseghin P, Mariotti J, Morabito L, et al. Donor lymphocyte infusion after allogeneic stem cell transplantation. Transfus Apher Sci. (2016) 54:345-55. doi: 10.1016/j.transci.2016.05.011

23. Rambaldi A, Biagi E, Bonini C, Biondi A, Introna M. Cell-based strategies to manage leukemia relapse: efficacy and feasibility of immunotherapy approaches. Leukemia. (2015) 29:1-10. doi: 10.1038/leu.2014.189

24. Yan CH, Wang JZ, Liu DH, Xu LP, Chen H, Liu KY, et al. Chemotherapy followed by modified donor lymphocyte infusion as a treatment for relapsed acute leukemia after haploidentical hematopoietic stem cell transplantation without in vitro T-cell depletion: superior outcomes compared with chemotherapy alone and an analysis of prognostic factors. Eur J Haematol. (2013) 91:304-14. doi: 10.1111/ejh.12168

25. Kochenderfer JN, Dudley ME, Kassim SH, Somerville R PT, Carpenter RO, Stetler-Stevenson $\mathrm{M}$, et al. Chemotherapy-refractory diffuse large B-cell lymphoma and indolent B-cell malignancies can be effectively treated with autologous $\mathrm{T}$ cells expressing an anti-CD19 chimeric antigen receptor. J Clin Oncol. (2015) 33:540-9. doi: 10.1200/JCO.2014.5 6.2025

26. Gill S, Tasian SK, Ruella M, Shestova O, Li Y, Porter DL, et al. Preclinical targeting of human acute myeloid leukemia and myeloablation using chimeric antigen receptor-modified T cells. Blood. (2014) 123:234354. doi: 10.1182/blood-2013-09-529537
27. Ghosh A, Smith M, James SE, Davila ML, Velardi E, Argyropoulos KV, et al. Donor CD19 CAR T cells exert potent graft-versus-lymphoma activity with diminished graft-versus-host activity. Nat Med. (2017) 23:2429. doi: $10.1038 / \mathrm{nm} .4258$

28. Magnani CF, Biondi A, Biagi E. Donor-derived CD19-targeted $\mathrm{T}$ cells in allogeneic transplants. Curr Opin Hematol. (2015) 22:497-502. doi: 10.1097/MOH.0000000000000178

29. Anwer F, Al-Aman S, Umar Z, Husnain M, McBride A, Persky D, et al. Donor origin CAR T cells: graft versus malignancy effect without GVHD, a systematic review. Immunotherapy. (2017) 9:123-30. doi: 10.2217/imt-2016-0127

30. Liu J, Zhong JF, Zhang X, Zhang C. Allogeneic CD19-CAR-T cell infusion after allogeneic hematopoietic stem cell transplantation in B cell malignancies. J Hematol Oncol. (2017). 10:35. doi: 10.1186/s13045-017-0405-3

31. Kolb H-J. Graft-versus-leukemia effects of transplantation and donor lymphocytes. Blood. (2008) 112:4371-83. doi: 10.1182/blood-2008-03-077974

32. Frey NV, Porter DL. Graft-versus-host disease after donor leukocyte infusions: presentation and management. Best Pract Res Clin Haematol. (2008) 21:20522. doi: 10.1016/j.beha.2008.02.007

33. Mirzaei HR, Pourghadamyari H, Rahmati M, Mohammadi A, Nahand JS, Rezaei A, et al. Gene-knocked out chimeric antigen receptor (CAR) T cells: tuning up for the next generation cancer immunotherapy. Cancer Lett. (2018) 423:95-104. doi: 10.1016/j.canlet.2018.03.010

34. Sommermeyer D, Hill T, Shamah SM, Salter AI, Chen Y, Mohler KM, et al. Fully human CD19-specific chimeric antigen receptors for T-cell therapy. Leukemia. (2017) 31:2191-9. doi: 10.1038/leu.2017.57

35. Brudno JN, Kochenderfer JN. Recent advances in CAR T-cell toxicity: mechanisms, manifestations and management. Blood Rev. (2019) 34:4555. doi: 10.1016/j.blre.2018.11.002

36. Hay KA, Hanafi LA, Li D, Gust J, Liles WC, Wurfel MM, et al. Kinetics and biomarkers of severe cytokine release syndrome after CD19 chimeric antigen receptor-modified T-cell therapy. Blood. (2017) 130:2295306. doi: 10.1182/blood-2017-06-793141

Conflict of Interest: NM was employed by the company Shanghai Genbase Biotechnology Co., Ltd.

The remaining authors declare that the research was conducted in the absence of any commercial or financial relationships that could be construed as a potential conflict of interest.

Copyright (C) $2020 \mathrm{Liu}, \mathrm{Liu}, \mathrm{Lyu}, \mathrm{Lu}$, Cui, Wang, Li, Mou, Deng and Yang. This is an open-access article distributed under the terms of the Creative Commons Attribution License (CC BY). The use, distribution or reproduction in other forums is permitted, provided the original author(s) and the copyright owner(s) are credited and that the original publication in this journal is cited, in accordance with accepted academic practice. No use, distribution or reproduction is permitted which does not comply with these terms. 